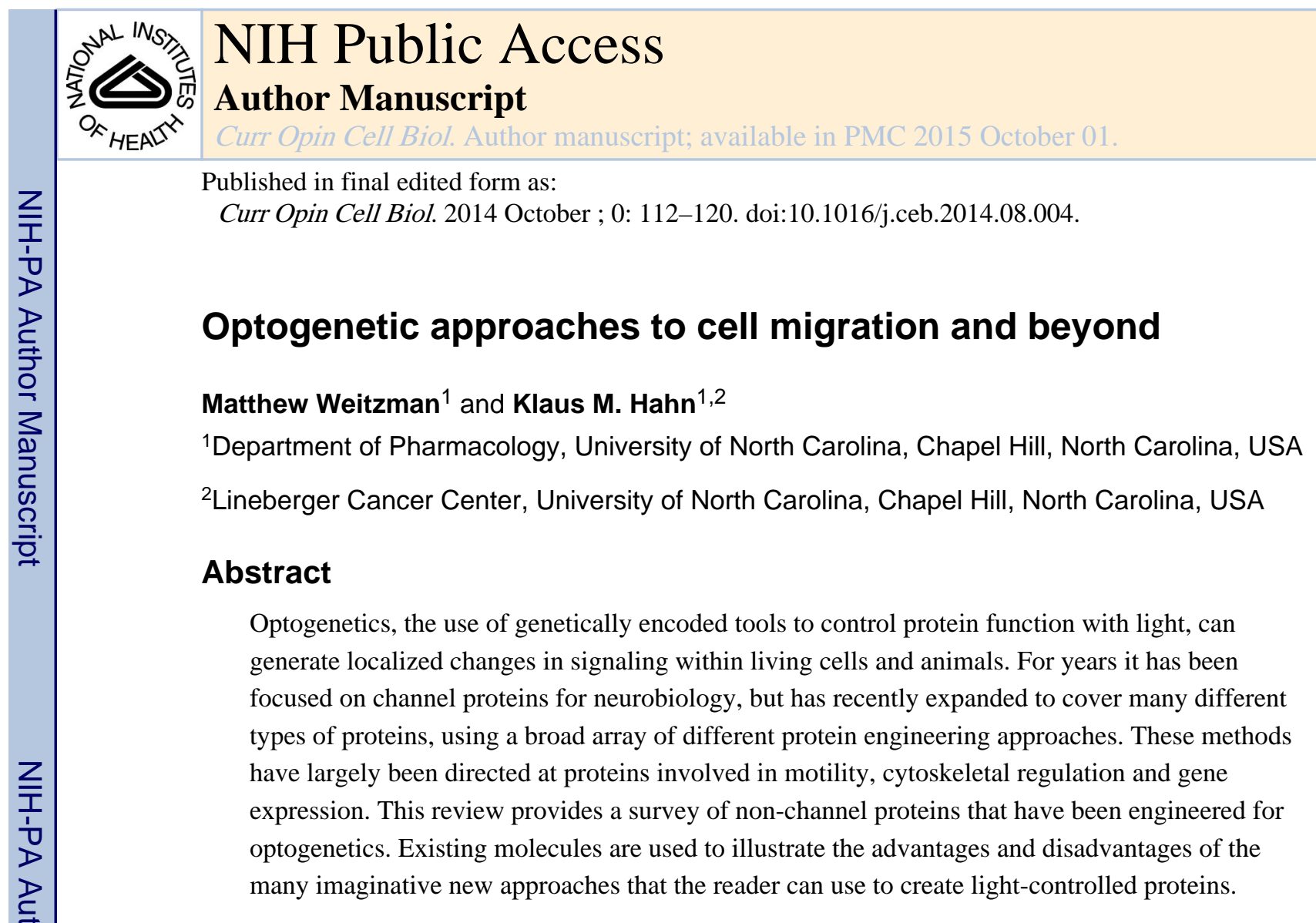

\title{
Introduction
}

Optogenetics refers to the control of cellular activity by genetically encoded tools that respond to light. In less than five years, non-channel optogenetics has expanded from a handful of applications to an impressive array of tools that control diverse proteins. Not only are many new molecules subject to photocontrol, but they have been produced through an impressive variety of approaches. Light has been used to induce/block protein interactions, control protein conformation, stimulate DNA binding, regulate enzyme activity, induce protein degradation and control subcellular targeting, all of which came about by creating tools based on natural photoreceptors. These photoreceptors contain a chromophore that absorbs a photon, resulting in the formation, destruction or isomerization of a chemical bond, which in turn causes a conformational change in the receptor. Typically the lightinduced changes in a chromophore-containing "sensory module" are transmitted to a different module that undergoes light-induced effector binding [1]. Due to this modularity, it is possible to engineer novel light regulated tools by linking natural photo-sensing modules to intrinsically light-insensitive effector proteins [2].

Prior reviews have covered the structure and photochemistry of photoreceptors [3-5], and there are very valuable papers that focus on optogenetic design without application in vivo. We focus here on optogenetics applied in live cells and animals, with emphasis on proteins

\footnotetext{
(C) 2014 Elsevier Ltd. All rights reserved.

Publisher's Disclaimer: This is a PDF file of an unedited manuscript that has been accepted for publication. As a service to our customers we are providing this early version of the manuscript. The manuscript will undergo copyediting, typesetting, and review of the resulting proof before it is published in its final citable form. Please note that during the production process errors may be discovered which could affect the content, and all legal disclaimers that apply to the journal pertain.
} 
involved in motility. Unfortunately, we cannot discuss the multiple exciting applications controlling gene expression, except to illustrate engineering approaches. Each photoreceptor is addressed in turn, with its uses in different designs for motility research (Figures 1-4).

\section{Light-Oxygen-Voltage domains}

Light-Oxygen-Voltage (LOV) domains have proven to be versatile light sensing modules. These relatively small domains $(\sim 12 \mathrm{kDa})$ respond to blue light $(\sim 400-500 \mathrm{~nm})$ and belong to the Per-ARNT-Sim protein family [4]. Within microseconds of photon absorption a covalent bond forms between a flavin co-factor and a conserved cysteine residue in the LOV domain [2, 6-8]. This covalent bond results in a conformational change, causing dissociation and unfolding of a C-terminal a-helix (Ja) $[6,9,10]$. In the absence of blue light, the flavincysteine bond is hydrolyzed, allowing $\mathrm{Ja}$ to refold and re-associate with the LOV domain [11]. This reversible, photo-induced unwinding of the terminal helix has been harnessed in a variety of ways to control protein function.

The LOV domain was used in living cells to control the activity of Rac1, a protein that induces cell protrusion and movement. Wu et al. fused Rac1 to the C-terminal Ja helix of the LOV domain to produce photoactivatable Rac1 (PA-Rac1) [12, 13]. In the dark the helix was tightly coiled, holding LOV near Rac1 and sterically blocking Rac1 interactions (Fig 1A). Upon irradiation, the helix unwound, leaving LOV attached to Rac1 via a long tether, freeing Rac1 to interact with its effectors. Rac1 could be reversibly and repeatedly activated in living cells, demonstrating that localized Rac1 activity was sufficient to produce precisely localized cell protrusion, and that Rac1 gradients alone could control directed motility. PARac1 provided to be a versatile tool for directing the motility of cells within living animals, elucidating collective migration in Drosophila embryos and neutrophil motility in zebrafish $[14,15]$. In living mice, expression of PA-Rac1 in the nucleus accumbens region of the brain allowed researchers to verify that cocaine-induced down regulation of Rac1 activity is required for reward effects during cocaine addiction. [16]. The simple steric blocking mechanism of PA Rac1 held promise for other GTPases, but it was discovered that the steric inhibition was dependent on a weak binding interaction between Rac1 and LOV. Analysis of the binding site is leading to new analogs where this approach is used for different families of molecules.

Simple steric inhibition of binding sites by the LOV domain has been more broadly useful for caging bio-active peptides (Fig 1B). Importantly, such 'caged peptides' can be used to control the activity of endogenous protein (see conclusion). Peptides have been appended to the end of the helix where light-induced unwinding of the helix freed them to interact with targets. Fragments of formin autoinhibitory domains that activate endogenous mDia1 [17, 18], an inhibitor of protein kinase A, and a myosin light chain kinase inhibitor [19] have been caged using this approach. Molecular modeling was used to incorporate residues from the vinculin-binding peptide IPA at several different places in the Ja helix, at positions where they did not interfere with helix-LOV binding, but were capable of interacting with vinculin once the helix unwound [20]. 
When proteins are fused to LOV, conformational changes in LOV can affect the attached protein (Fig 3c). Strickland et al's "LOV-TAP" system provided an important proof of principle in vitro [21]. When an E. Coli trp repressor was fused to LOV, its DNA affinity increased five fold in the light. The trp domain was attached to the terminus of the Ja helix so that the domain was perturbed when Ja was in its LOV-bound, dark state conformation. Introduction of point mutations further stabilized LOV-Ja docking, increasing the difference in DNA affinity from 5-fold to 70-fold [22]. Moglich et al. replaced the chemosensory domain of histidine kinase with a LOV domain, thereby generating a kinase activated by light rather than oxygen, and used it to drive gene expression in E. Coli [23].

Finally, light-induced heterodimerization has proven to be a very powerful and versatile approach (Fig 2). Strickland et al. reported tunable light-inducible dimerization tags (TULIPs) (Fig 2B) [24]. LOV2 domain from Avena sativa phototropin 1 was used to sterically "cage" a peptide that bound to an engineered PDZ domain (ePDZ), using a peptide caging approach like that described above. The caged peptide was anchored at the plasma membrane, and the ePDZ domain was attached to proteins that were active only at the plasma membrane. Irradiation freed the peptide to interact with the ePDZ domain, leading to movement of the protein to its site of action. Mechanistic studies showed that the rate of return to the dark state was determined not by release of the peptide-Ja fusion from the ePDZ domain, but by the rate at which the LOV domain returned to the dark state.

Strickland et al. asked whether spatially constrained activation of Cdc 42 was sufficient for polarized growth in budding yeast [25]. Global recruitment of Cdc 42 to the entire plasma membrane caused growth arrest, but localized recruitment could specify the direction of polarized growth and the orientation of mating projections.

Yazawa et al. used light-induced interaction of the proteins GIGANTEA and LOVcontaining FKF1 to control transcription factors, and to recruit Rac1 to the plasma membrane (similar to cases shown in Fig 2)[26]. Likely due to the slow return of this system to the monomeric state, Rac1 remained on the membrane for at least 1.5 hours after brief exposure to activating light. More recent applications exploit LOVs rapid kinetics when controlling gene transcription. They use VIVID, the smallest LOV-containing protein [27], and EL222 [28], a bacterial LOV-containing protein with light-dependent DNA binding.

Several advantages have contributed to the extensive use of LOV domains. The lightinduced conformational change is very fast (complete within $\sim 4$ microseconds [6]) and point mutations can be used to adjust the rate of return to the dark state. Christie et al. employed random mutagenesis to identify amino acid residues involved in photoswitching, primarily specific side chains near the cysteinyl-flavin adduct [8]. In studies of the slow cycling fungal LOV photoreceptor (VVD), Zoltowski et al. showed that substitution of active site residues could alter half lives from $\sim 30$ seconds to $>48$ hours [29]. The LOV domain has advantages for animal studies in that the flavin cofactor is incorporated simply upon expression, in species from C. elegans to mice, and control is achieved simply by turning the light on and off. 


\section{Phytochromes}

Phytochromes are light sensitive proteins that modulate seed germination and shade avoidance [30, 31]. Phytochrome B (PhyB) and the transcription factor phytochrome interaction factor (PIF3) heterodimerize when irradiated with red light. Unlike other photoproteins used in optogenetic tools, different wavelengths are used to induce dimerization versus monomerization (dimerization: $\sim 650 \mathrm{~nm}-670 \mathrm{~nm}$; monomerization: $\sim 700 \mathrm{~nm}-750 \mathrm{~nm}$ ) [32]. PhyB photoswitching occurs through photoisomerization of a tetrapyrrole chromophore such as phycocyanobilin (PCB) that is not normally found in mammalian systems, so the chromophore must be added to culture media. However, NIH3T3 cells have shown rapid autoligation of externally supplied PCB chromophore, providing fluorescence within 30min of exposure to 5mM PCB [30].

Levskaya et al. activated the transcriptional activity of PIF3 through light-induced PIF3PhyB binding, and recruited Rho guanine nucleotide exchange factors (GEFs) to the plasma membrane to locally activate GTPases for control of cell morphology (similar to cases shown in Fig 2) [30]. Leung et al. used PhyB-PIF interaction to induce interaction between Wiskott Aldrich Syndrome protein (WASP) and its upstream activator Cdc42 [33]. To prevent interactions before irradiation, they used a Cdc42 mutant that remained always in the inactive conformation. The forced dimerization of this mutant with WASP was sufficient to induce WASP activation. Toettcher et al. showed that recruitment can be titrated to specific levels by using different ratios of 650 and 750nm light [34]. Cells express optogenetic components at varying levels, and signaling circuits vary over time; this was overcome by using live cell readings of intracellular activation to update irradiation levels in real time. This photoreceptor has been applied in several different systems for control of gene expression [35, 36], including the use of split transcription factors [37].

More recently, Yang et al. used PhyB constructs anchored to different subcellular targets to generate a fast, reversible and titratable system for manipulation of protein localization in budding yeast (Fig 2C)[38]. Toettcher et al used similar tactics to design their opto-SOS system. Constitutively active SOS was targeted to the plasma membrane, where it activated RAS. Both the frequency and duration of RAS activation could be precisely controlled, elucidating how a single pathway could specify distinct physiological outcomes [39].

Rather than harness Phy/PIF dimerization, Gasser et al. demonstrated a unique application of this protein family. Light-induced conformational changes of Phy affected the conformation of attached proteins (similar to Fig 3C). Based on striking similarities in structure between bacterial phytochromes and human phosphodiesterases, the photoresponsive domain of bacterial phytochrome was combined with the effector module of human phosphodiesterase 2A [40]. This led to "LAPD" (light-activated phosphodiesterase), which hydrolyzed cAMP and cGMP in response to red light, in both eukaryotic cell culture and zebrafish embryos. Notably, endogenous biliverdin cofactor present in $\mathrm{CHO}$ cells was sufficient for maximal light response; exogenously added cofactor had no effect on LAPD activity [40]. 


\section{Cryptochromes}

Cryptochrome (Cry) proteins are blue light absorbing plant photosensors that heterodimerize with the transcripton factor CIB1 upon irradiation [14]. Cry proteins have an N-terminal DNA photolyase homology region (PHR) that binds a flavin adenine dinucleotide chromophore, and a C-terminal domain essential for signal transduction [41]. Like the LOV proteins, Cry proteins incorporate the flavin cofactor simply upon protein expressions. Kennedy et al. modified the two proteins to make them practical for optogenetic applications (Fig 2C)[41]. Punctate perinuclear structures formed by CIB1 were eliminated by removing a conserved helix-loop-helix domain involved in dimerization and DNA binding, to form a so-called CIBN fragment. The PHR domain alone was just as efficient as full length Cry in targeting fluorescent proteins to the plasma membrane. Heterodimerization was rapid, but the return to the dark state was substantially slower than LOV and Phy/PIF, with a half life of $\sim 5.5 \mathrm{~min}$. The system was used for light activated reconstitution of split proteins, to regenerate Gal4 transcription factor and Cre recombinase activity. It has proven to be a robust tool, applied in multiple applications. Other designs have been used to control gene activity in zebrafish [42], and in mice through epigenetic modifications [43].

Cry2-Cib dimerization has been used to control membrane lipid composition. By bringing phosphatidylinositol 5-phosphatase to the plasma membrane, Idevall-Hagren et al. demonstrated reversible and rapid control of local phosphoinositide levels (similar to Fig 2C)[44]. Kakumoto et al. were able to induce acute local phosphatidylinositol $(3,4,5)$ triphosphate (PIP3) signaling using a phosphoinositide 3-kinase (PI3K) photoswitch [45]. CIBN was anchored to the plasma membrane and the PHR domain of CRY2 was fused to the regulatory subunit of PI3K. Irradiation led to translocation of the regulatory subunit to the plasma membrane, where it associated with endogenous p110, the catalytic subunit of PI3K, triggering PIP3 production and resulting in the formation of filopodia and lamellipodia [46].

In similar fashion, O'Neill et al. utilized Cry2-Cib heterodimerizaiton to control the activity of endogenous heterotrimeric $\mathrm{G}$ protein $\alpha \beta \gamma$ subunits in motile immune cells [47]. This was achieved by coexpressing a membrane anchored CIBN construct with a Cry2-RGS4 (Regulator of G protein signaling 4) fusion in the RAW 264.7 macrophage cell line. Upon light stimulation, CIBN recruited CRY2-RGS4 to the plasma membrane, leading to localized increase in GAP activity on endogenous ai and aq subunits. Importantly, the spatial control afforded by light allowed O'Neill et al. to show that a gradient of active G protein ai and $\beta \gamma$ subunits was sufficient to generate directed cell migration [47].

Recently, Cry2 was shown to homo-oligomerize in response to light, providing another avenue for design of optogenetic tools (Fig 3B). Bugaj et al. found that the Cry2 PHR domain, expressed alone in mammalian cells, formed puncta that disappeared with minutes after light was removed [48]. Puncta size and number increased sigmoidally over time and, consistent with Kennedy et al., decayed with a half life of $\sim 5.5 \mathrm{~min}$. This Cry2 oligomerization could be used to activate $\beta$-catenin through light-induced oligomerization of the upstream protein LRP6. Cry2 homo-oligomerization was also used to drive Rac1 and RhoA activation upon translocation to the plasma membrane and vesicles, leading to 
membrane retraction. Illumination of fibroblasts expressing Cry2-RhoA led to stress fibers, and Cry2-RhoA clusters colocalized with the fibers.

Kim et al., exploited Cry2 interactions to engineer an optically controllable Fibroblast growth factor receptor system termed optoFGFR1 (Fig 2A). The Cry2 PHR domain was linked to the cytoplasmic region of human FGFR1 and anchored in the membrane. Blue light induced dimerization and trans-autophosphorylation, leading to activation of FGFR signaling. This simple system was shown to effectively control cell polarity and induce directed cell migration with high spatial and temporal resolution [49].

Wend et al directly compared CRY2 homooligomerization with Cry2-Cib heterodimerization when designing a photocontrolled version of the kinase C-RAF [50]. Light mediated activation of C-RAF-CRY2 alone or in combination with C-RAF-CIBN, was evaluated by quantifying downstream ERK1/2 phosphorylation. Cells expressing CRAF-CRY2 alone produced much more Erk phosphorylation than did the heterodimeric pair. The authors propose that CRY2 homodimerization produces a more favorable geometry for the Raf dimerization that is required for activity.

In a unique approach to light-induced inhibition, Lee et al attached CIB 1 to multiple subunits of a large multimeric complex [51]. Upon irradiation, proteins bearing Cry 2 were bound to the complex, reversibly sequestering them away from their site of activity. $\mathrm{Ca} 2+$ / calmodulin-dependent protein kinase IIa (CaMKIIa) self assembles into oligomers containing twelve identical subunits. This was used as the sequestering scaffold, with each CaMKIIa subunit fused to a CIB1 molecule. After Cry2 bound to the complex, its homodimerization led to the formation of larger order complexes whose number and size could be controlled by the intensity and number of light pulses. This "LARIAT " system (light-activated reversible inhibition by assembled trap) was shown to effectively and reversibly sequester fluorescent proteins, and was used to inhibit tubulin, Vav2, Tiam1, Rac1, RhoG, and Cdc42. In all cases, inactivation of these proteins led to membrane retraction. Cleverly, the authors did not directly link all of these proteins to Cry2, but instead fused Cry 2 to an anti-GFP antibody fragment, enabling sequestration of GFP-fused proteins (Fig 3A). In addition, clusters could be repeatedly assembled, were formed within seconds, dispersed within minutes after withdrawal of light and could be used for localized inhibition. To date, this system has been used to reverse the effects of target protein overexpression.

\section{Fluorescent proteins, ultraviolet-B photoreceptors and opsins}

Other photoresponsive proteins have untapped potential as optogenetic agents, including fluorescent proteins, whose photophysics are understood in great depth. Zhou et al. modified the fluorescent protein Dronpa to generate light modulated dimerization systems that could control protein localization and activity [52]. Monomeric Dronpa was generated through modification of a tetrameric parent. Its fluorescence switches off under cyan light ( 500nm) and on under violet light $(\sim 400 \mathrm{~nm})$. Switching occurs due to changes in a $\beta$ strand near the chromophore, in an area that is part of the dimerization surface of the parental tetramer, so conformational changes that occur during on/off switching could be engineered to affect monomer-dimer equilibrium. Mutagenesis of Dronpa culminated in a simple design called 
fluorescent light-inducible proteins (FLIPs), capable of targeting proteins to the plasma membrane and to sterically cage protein activity (Fig 1C). FLIPs serve as their own fluorescent reporters. Zhou et al. demonstrated the caging potential of FLIPs by controlling the Cdc42 RhoGEF intersectin. Dronpa variants were fused to the $\mathrm{N}$ - and C-termini of the intersectin Dbl homology (DH) domain such that dimerization of Dronpa masked important binding surfaces. Light mediated dissociation allowed the DH domain to bind and activate endogenous Cdc42. FLIP-intersectin constructs were able to robustly induce precisely localized filopodia and lamellipodia. FLIP-intersectin was used to demonstrate that Cdc42 activation can lengthen existing filopodia; $\mathrm{Cdc} 42$ effectors which extend rather than initiate filopodia formation had not been previously identified.

Arabidopsis thaliana has provided us with UVR8, a member of the ultraviolet B photoreceptor family [53]. The ultraviolet B light to which this photoreceptor responds $(280-315 \mathrm{~nm})$ is difficult for cells to tolerate and necessitates the use of specialized microscopy equipment (these wavelengths are not effectively transmitted by glass) [54]. However, the receptor can be selectively activated in the same cells as the other photoreceptors described above, so may have a niche in multiplexed activation studies. In the absence of UV light, UVR8 receptors form homodimers. The interface between the proteins is rich in the tryptophan residues that act as chromophores. Irradiation induces an irreversible change in conformation, leading to monomerization of UVR8. In Arabidopsis, monomerization enables binding to the E3 ubiquitin ligase COP1 [55]. Crefcoeur et al. took advantage of this naturally evolved binding pair to control transcription (Fig 2D)[54]. The transcriptional activation domain of NF- $\kappa$ B was fused to UVR8, and the GAL4 DNA binding domain to COP1. Binding was induced using a second light path that did not rely on microscope optics. Crefcoeur et al. engineered such a second light path based on a 290$310 \mathrm{~nm}$ LED and a collimator that uses silicon wafers rather than optical lenses, enabling simultaneous light stimulation and observation. Chen et al. used the UVR8 photoreceptor to produce a light triggered protein secretion system [56]. Multiple copies of YFP-UVR8 were fused to the C-terminal intracellular domain of vesicular stomatitis virus glycoprotein (VSVG). In the absence of UV-B light, UVR8 homodimerization led to higher order oligomerization, causing the VSVG construct to be trapped in the ER. A seven second exposure to UV-B light disrupted VSVG oligomerization, enabling trafficking through the Golgi apparatus and cell surface expression. Surface expression was seen within thirty minutes and the Golgi complex was nearly empty after two hours.

Opsins, light-sensitive members of the G protein superfamily, undergo a light-induced conformational change resulting in activation of the Ga subunit. Human color opsins, coupled to Gatc subunits in retinal photoreceptor cells, have been reengineered to control $\mathrm{Gi} / \mathrm{o}, \mathrm{Gq}$ and Gs signaling in other mammalian cells [57]. The blue opsins were used to precisely steer the migration of immune cells through activation of endogenous $\mathrm{G}$ proteins [58]. Rod opsins have been used similarly, to activate Gas[59].

\section{Conclusion}

The optogenetic tools described above can be roughly grouped into five basic approaches. These are (1) light-induced dimerization, for targeting proteins to specific locations or 
bringing proteins together to generate activity, (2) steric blocking of active sites, for whole proteins or for bioactive peptides, (3) sequestration, where molecules are held at locations away from their active site upon irradiation, (4) photoreceptor-target fusions where receptor conformation affects the target and (5) protein activity regulated by light induced oligomerization.

These designs influence the prevalence of potential artifacts. In some methods an analog of the target protein, made sensitive to light, is added to the cell. This can be problematic when the goal is to inhibit the protein; the analog is most easily used by overexpressing it, thereby enabling light-induced elimination of overexpression effects. Alternately, endogenous protein could be knocked down and rescued with the photo-inhibitable analog. Protein analogs that are activated by light can act as dominant negative analogs prior to irradiation, unless interactions with upstream regulators are knocked out. In such cases one must be careful that these interactions do not participate in targeting. Tools used to modulate the activity of endogenous proteins are not subject to these issues. However, they may be prevented from accessing their targets due to competition from native protein ligands. Achievement of high affinity in these cases can be very important.

The photoreceptors are in equilibrium between an 'on' and an 'off' state. Even in the dark, a small, equilibrium amount of activated material is present [60,61]. This 'leakiness' requires a careful adjustment of protein expression levels, and its importance will depend on how sensitive the cell is to small changes in protein activity, to the difference between on and off state activity levels of the optogenetic tool, and the extent to which the design produces small amounts of active material in the dark. It can be useful to use fluorescent tags on the optogenetic proteins, to roughly determine expression levels (eg using average brightness for flat cells). Appropriate expression levels can be determined and used to generate stable cell lines selected by FACS.

All the photoreceptors used here are activated in well under a second. Although for some approaches biological activation also depends on diffusion of an active species, this has not been a serious limitation. More important is the slow rate at which some photoreceptors return to the dark state. The Phy/Pif system has very fast return kinetics. Fast mutants of LOV can return with a half-life of roughly seven seconds. Others receptors require minutes or are irreversible. A slow return to the dark state can be advantageous, especially for prolonged activation. With slow return to the dark state, activation can be maintained with short pulses of light at regular intervals, reducing phototoxcity.

Spatial control is limited by the resolution of the light microscope (determining the minimal irradiation area), and the rate of diffusion relative to the rate of return to the dark state ([12] supplementary data). The Phy/Pif system has superb spatial resolution, because both on and off rates are rapid. Different regions of the cell can be continuously bathed in either the activating or inactivating wavelengths, so that molecules which move across the border are rapidly converted to the alternate form.

The unprecedented spatio-temporal control provided by optogenetics is enabling us to test models of motility that include rapid organelle and cell dynamics long thought to be 
important, but beyond the reach of experimentation. The wavelength palette already offered by photoreceptors and fluorescent proteins will make it possible to visualize and manipulate multiple molecules in the same cell. The future is bright. We are fortunate to have so many different design approaches available for what will surely be completely unexpected optogenetic applications and opportunities.

\section{Acknowledgments}

We are grateful to Onur Dagliyan and Hui Wang for critical reading of the manuscript, and thank the NIH for financial support (GM103723 and GM102924).

\section{References}

1. Lim WA. The modular logic of signaling proteins: building allosteric switches from simple binding domains. Current opinion in structural biology. 2002; 12:61-68. [PubMed: 11839491]

2. Yin T, Wu YI. Guiding lights: recent developments in optogenetic control of biochemical signals. Pflugers Archiv : European journal of physiology. 2013; 465:397-408. [PubMed: 23417571]

3. Moglich A, Yang X, Ayers RA, Moffat K. Structure and function of plant photoreceptors. Annual review of plant biology. 2010; 61:21-47.

4. Moglich A, Ayers RA, Moffat K. Structure and signaling mechanism of Per-ARNT-Sim domains. Structure. 2009; 17:1282-1294. [PubMed: 19836329]

5. Losi A, Gartner W. The evolution of flavin-binding photoreceptors: an ancient chromophore serving trendy blue-light sensors. Annual review of plant biology. 2012; 63:49-72.

6. Swartz TE, Corchnoy SB, Christie JM, Lewis JW, Szundi I, Briggs WR, Bogomolni RA. The photocycle of a flavin-binding domain of the blue light photoreceptor phototropin. The Journal of biological chemistry. 2001; 276:36493-36500. [PubMed: 11443119]

7. Schleicher E, Kowalczyk RM, Kay CW, Hegemann P, Bacher A, Fischer M, Bittl R, Richter G, Weber S. On the reaction mechanism of adduct formation in LOV domains of the plant blue-light receptor phototropin. Journal of the American Chemical Society. 2004; 126:11067-11076. [PubMed: 15339193]

8. Christie JM, Corchnoy SB, Swartz TE, Hokenson M, Han IS, Briggs WR, Bogomolni RA. Steric interactions stabilize the signaling state of the LOV2 domain of phototropin 1. Biochemistry. 2007; 46:9310-9319. [PubMed: 17658895]

9. Harper SM, Neil LC, Gardner KH. Structural basis of a phototropin light switch. Science. 2003; 301:1541-1544. [PubMed: 12970567]

10. Halavaty AS, Moffat K. N- and C-terminal flanking regions modulate light-induced signal transduction in the LOV2 domain of the blue light sensor phototropin 1 from Avena sativa. Biochemistry. 2007; 46:14001-14009. [PubMed: 18001137]

11. Kasahara M, Swartz TE, Olney MA, Onodera A, Mochizuki N, Fukuzawa H, Asamizu E, Tabata S, Kanegae H, Takano M, et al. Photochemical properties of the flavin mononucleotide-binding domains of the phototropins from Arabidopsis, rice, and Chlamydomonas reinhardtii. Plant physiology. 2002; 129:762-773. [PubMed: 12068117]

12. Wu YI, Frey D, Lungu OI, Jaehrig A, Schlichting I, Kuhlman B, Hahn KM. A genetically encoded photoactivatable Rac controls the motility of living cells. Nature. 2009; 461:104-108. [PubMed: 19693014]

13. Wu YI, Wang X, He L, Montell D, Hahn KM. Spatiotemporal control of small GTPases with light using the LOV domain. Methods in enzymology. 2011; 497:393-407. [PubMed: 21601095]

14. Wang X, He L, Wu YI, Hahn KM, Montell DJ. Light-mediated activation reveals a key role for Rac in collective guidance of cell movement in vivo. Nature cell biology. 2010; 12:591-597.

15. Yoo SK, Deng Q, Cavnar PJ, Wu YI, Hahn KM, Huttenlocher A. Differential regulation of protrusion and polarity by PI3K during neutrophil motility in live zebrafish. Developmental cell. 2010; 18:226-236. [PubMed: 20159593] 
16. Dietz DM, Sun H, Lobo MK, Cahill ME, Chadwick B, Gao V, Koo JW, Mazei-Robison MS, Dias $\mathrm{C}$, Maze I, et al. Rac1 is essential in cocaine-induced structural plasticity of nucleus accumbens neurons. Nature neuroscience. 2012; 15:891-896.

17. Baarlink C, Wang H, Grosse R. Nuclear actin network assembly by formins regulates the SRF coactivator MAL. Science. 2013; 340:864-867. [PubMed: 23558171]

18. Rao MV, Chu PH, Hahn KM, Zaidel-Bar R. An optogenetic tool for the activation of endogenous diaphanous-related formins induces thickening of stress fibers without an increase in contractility. Cytoskeleton. 2013; 70:394-407. [PubMed: 23677607]

19. Yi JJ, Wang H, Vilela M, Danuser G, Hahn KM. Manipulation of Endogenous Kinase Activity in Living Cells Using Photoswitchable Inhibitory Peptides. ACS synthetic biology. 2014

20. Lungu OI, Hallett RA, Choi EJ, Aiken MJ, Hahn KM, Kuhlman B. Designing photoswitchable peptides using the AsLOV2 domain. Chemistry \& biology. 2012; 19:507-517. [PubMed: 22520757]

21. Strickland D, Moffat K, Sosnick TR. Light-activated DNA binding in a designed allosteric protein. Proceedings of the National Academy of Sciences of the United States of America. 2008; 105:10709-10714. [PubMed: 18667691]

22. Strickland D, Yao X, Gawlak G, Rosen MK, Gardner KH, Sosnick TR. Rationally improving LOV domain-based photoswitches. Nature methods. 2010; 7:623-626. [PubMed: 20562867]

23. Moglich A, Ayers RA, Moffat K. Design and signaling mechanism of light-regulated histidine kinases. Journal of molecular biology. 2009; 385:1433-1444. [PubMed: 19109976]

24. Strickland D, Lin Y, Wagner E, Hope CM, Zayner J, Antoniou C, Sosnick TR, Weiss EL, Glotzer M. TULIPs: tunable, light-controlled interacting protein tags for cell biology. Nature methods. 2012; 9:379-384. [PubMed: 22388287]

25. Park HO, Bi E. Central roles of small GTPases in the development of cell polarity in yeast and beyond. Microbiology and molecular biology reviews : MMBR. 2007; 71:48-96. [PubMed: 17347519]

26. Yazawa M, Sadaghiani AM, Hsueh B, Dolmetsch RE. Induction of protein-protein interactions in live cells using light. Nature biotechnology. 2009; 27:941-945.

27. Wang X, Chen X, Yang Y. Spatiotemporal control of gene expression by a light-switchable transgene system. Nature methods. 2012; 9:266-269. [PubMed: 22327833]

28. Rivera-Cancel G, Motta-Mena LB, Gardner KH. Identification of natural and artificial DNA substrates for light-activated LOV-HTH transcription factor EL222. Biochemistry. 2012; 51:10024-10034. [PubMed: 23205774]

29. Zoltowski BD, Vaccaro B, Crane BR. Mechanism-based tuning of a LOV domain photoreceptor. Nature chemical biology. 2009; 5:827-834.

30. Levskaya A, Weiner OD, Lim WA, Voigt CA. Spatiotemporal control of cell signalling using a light-switchable protein interaction. Nature. 2009; 461:997-1001. [PubMed: 19749742]

31. Quail PH. Phytochrome photosensory signalling networks. Nature reviews Molecular cell biology. 2002; 3:85-93.

32. Kendrick, RE.; Kronenberg, GHM. Photomorphogenesis in plants. Dordrecht ; Boston: Kluwer Academic; 1994.

33. Leung DW, Otomo C, Chory J, Rosen MK. Genetically encoded photoswitching of actin assembly through the Cdc42-WASP-Arp2/3 complex pathway. Proceedings of the National Academy of Sciences of the United States of America. 2008; 105:12797-12802. [PubMed: 18728185]

34. Toettcher JE, Gong D, Lim WA, Weiner OD. Light-based feedback for controlling intracellular signaling dynamics. Nature methods. 2011; 8:837-839. [PubMed: 21909100]

35. Shimizu-Sato S, Huq E, Tepperman JM, Quail PH. A light-switchable gene promoter system. Nature biotechnology. 2002; 20:1041-1044.

36. Muller K, Engesser R, Schulz S, Steinberg T, Tomakidi P, Weber CC, Ulm R, Timmer J, Zurbriggen MD, Weber W. Multi-chromatic control of mammalian gene expression and signaling. Nucleic acids research. 2013; 41:e124. [PubMed: 23625964]

37. Muller K, Engesser R, Metzger S, Schulz S, Kampf MM, Busacker M, Steinberg T, Tomakidi P, Ehrbar M, Nagy F, et al. A red/far-red light-responsive bi-stable toggle switch to control gene expression in mammalian cells. Nucleic acids research. 2013; 41:e77. [PubMed: 23355611] 
38. Yang X, Jost AP, Weiner OD, Tang C. A light-inducible organelle-targeting system for dynamically activating and inactivating signaling in budding yeast. Molecular biology of the cell. 2013; 24:2419-2430. [PubMed: 23761071]

39. Toettcher JE, Weiner OD, Lim WA. Using optogenetics to interrogate the dynamic control of signal transmission by the Ras/Erk module. Cell. 2013; 155:1422-1434. [PubMed: 24315106]

40. Gasser C, Taiber S, Yeh CM, Wittig CH, Hegemann P, Ryu S, Wunder F, Moglich A. Engineering of a red-light-activated human cAMP/cGMP-specific phosphodiesterase. Proc Natl Acad Sci U S A. 2014; 111:8803-8808. [PubMed: 24889611]

41. Kennedy MJ, Hughes RM, Peteya LA, Schwartz JW, Ehlers MD, Tucker CL. Rapid blue-lightmediated induction of protein interactions in living cells. Nature methods. 2010; 7:973-975. [PubMed: 21037589]

42. Liu H, Gomez G, Lin S, Lin S, Lin C. Optogenetic control of transcription in zebrafish. PloS one. 2012; 7:e50738. [PubMed: 23226369]

43. Konermann S, Brigham MD, Trevino AE, Hsu PD, Heidenreich M, Cong L, Platt RJ, Scott DA, Church GM, Zhang F. Optical control of mammalian endogenous transcription and epigenetic states. Nature. 2013; 500:472-476. [PubMed: 23877069]

44. Idevall-Hagren O, Dickson EJ, Hille B, Toomre DK, De Camilli P. Optogenetic control of phosphoinositide metabolism. Proceedings of the National Academy of Sciences of the United States of America. 2012; 109:E2316-2323. [PubMed: 22847441]

45. Kakumoto T, Nakata T. Optogenetic control of PIP3: PIP3 is sufficient to induce the actin-based active part of growth cones and is regulated via endocytosis. PloS one. 2013; 8:e70861. [PubMed: 23951027]

46. Welch CM, Elliott H, Danuser G, Hahn KM. Imaging the coordination of multiple signalling activities in living cells. Nature reviews Molecular cell biology. 2011; 12:749-756.

47. O’Neill PR, Gautam N. Subcellular optogenetic inhibition of G proteins generates signaling gradients and cell migration. Molecular biology of the cell. 2014; 25:2305-2314. [PubMed: 24920824]

48. Bugaj LJ, Choksi AT, Mesuda CK, Kane RS, Schaffer DV. Optogenetic protein clustering and signaling activation in mammalian cells. Nature methods. 2013; 10:249-252. [PubMed: 23377377]

49. Kim N, Kim JM, Lee M, Kim CY, Chang KY, Heo WD. Spatiotemporal Control of Fibroblast Growth Factor Receptor Signals by Blue Light. Chemistry \& biology. 2014; 21:903-912. [PubMed: 24981772]

50. Wend S, Wagner HJ, Muller K, Zurbriggen MD, Weber W, Radziwill G. Optogenetic control of protein kinase activity in mammalian cells. ACS synthetic biology. 2014; 3:280-285. [PubMed: 24090449]

51. Lee S, Park H, Kyung T, Kim NY, Kim S, Kim J, Heo WD. Reversible protein inactivation by optogenetic trapping in cells. Nature methods. 2014; 11:633-636. [PubMed: 24793453]

52. Zhou XX, Chung HK, Lam AJ, Lin MZ. Optical control of protein activity by fluorescent protein domains. Science. 2012; 338:810-814. [PubMed: 23139335]

53. Rizzini L, Favory JJ, Cloix C, Faggionato D, O’Hara A, Kaiserli E, Baumeister R, Schafer E, Nagy F, Jenkins GI, et al. Perception of UV-B by the Arabidopsis UVR8 protein. Science. 2011; 332:103-106. [PubMed: 21454788]

54. Crefcoeur RP, Yin R, Ulm R, Halazonetis TD. Ultraviolet-B-mediated induction of protein-protein interactions in mammalian cells. Nature communications. 2013; 4:1779.

55. Favory JJ, Stec A, Gruber H, Rizzini L, Oravecz A, Funk M, Albert A, Cloix C, Jenkins GI, Oakeley EJ, et al. Interaction of COP1 and UVR8 regulates UV-B-induced photomorphogenesis and stress acclimation in Arabidopsis. The EMBO journal. 2009; 28:591-601. [PubMed: 19165148]

56. Chen D, Gibson ES, Kennedy MJ. A light-triggered protein secretion system. The Journal of cell biology. 2013; 201:631-640. [PubMed: 23671313]

57. Karunarathne WK, Giri L, Kalyanaraman V, Gautam N. Optically triggering spatiotemporally confined GPCR activity in a cell and programming neurite initiation and extension. Proceedings of 
the National Academy of Sciences of the United States of America. 2013; 110:E1565-1574. [PubMed: 23479634]

58. Karunarathne WK, Giri L, Patel AK, Venkatesh KV, Gautam N. Optical control demonstrates switch-like PIP3 dynamics underlying the initiation of immune cell migration. Proceedings of the National Academy of Sciences of the United States of America. 2013; 110:E1575-1583. [PubMed: 23569254]

59. Bailes HJ, Zhuang LY, Lucas RJ. Reproducible and sustained regulation of Galphas signalling using a metazoan opsin as an optogenetic tool. PloS one. 2012; 7:e30774. [PubMed: 22292038]

60. Yao X, Rosen MK, Gardner KH. Estimation of the available free energy in a LOV2-J alpha photoswitch. Nature chemical biology. 2008; 4:491-497.

61. Moglich A, Moffat K. Engineered photoreceptors as novel optogenetic tools. Photochemical \& photobiological sciences : Official journal of the European Photochemistry Association and the European Society for Photobiology. 2010; 9:1286-1300. 
A
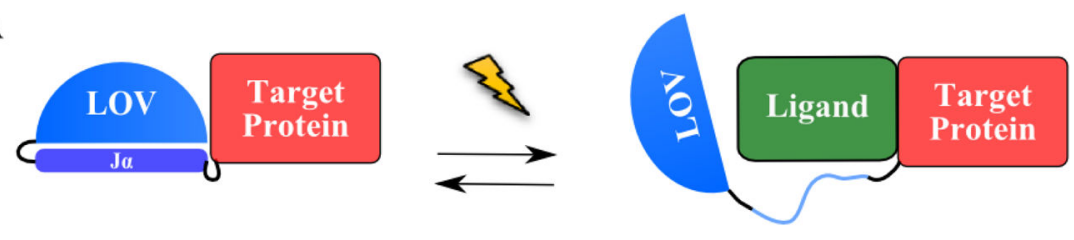

B

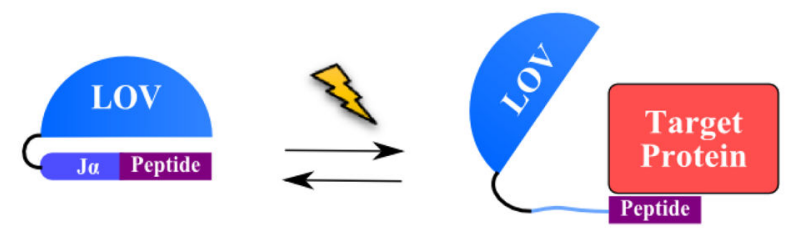

C

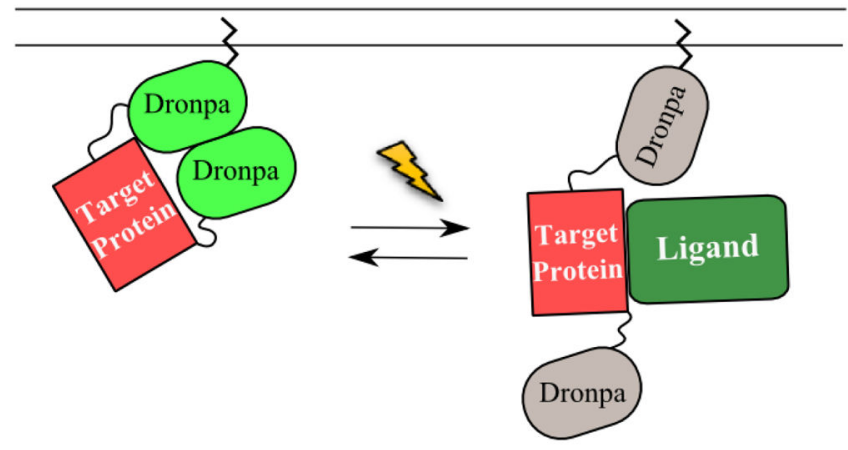

Figure 1.

Steric blocking of active sites. A. LOV blocks the target's active site when the Ja helix is tightly coiled, but when Ja unwinds to generate a long tether, the active site is exposed. B. A peptide that affects endogenous proteins can be appended to the Ja helix, where it is sterically blocked from interactions until LOV is irradiated. C. Dimerization of DRONPA across the active site of the targeted protein blocks interaction with ligands until irradiation disrupts dimerization. 
A

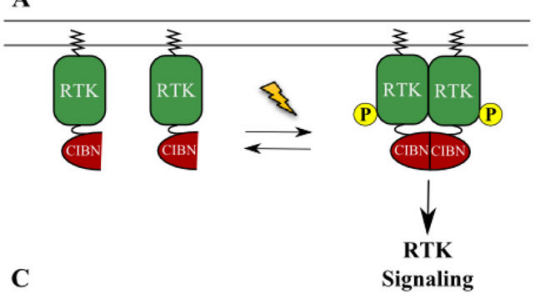

$\mathrm{C}$
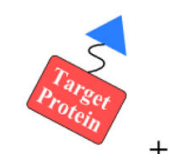

$+$

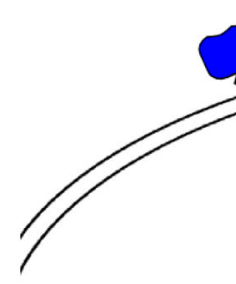

D

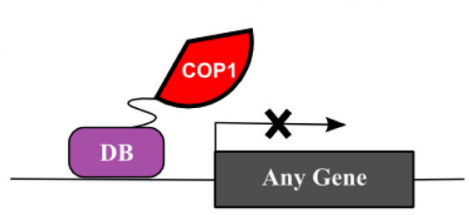

\section{TA}

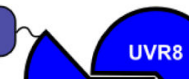

๑
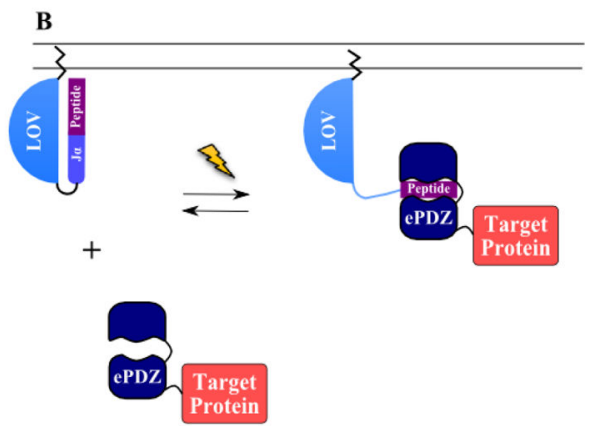

\section{(n}

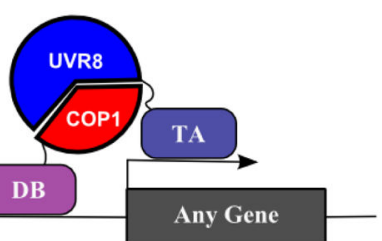

Figure 2.

Optogenetic Control by Dimerization. A. The optoFGFR1 system for dimerization-mediated activation of receptor tyrosine kinase (RTK) signaling[49]. B. The TULIP system for "dimerization"-mediated recruitment. C. Light-induced dimerization for targeting protein to sites of action, including the plasma membrane and organelles.. This has been driven by Phy/PIF, Cry/CIB and FKF1/GIGANTEA. D. Gene expression through the dimerization of UVR8 and COP1. COP1 is expressed as a fusion linked to a DNA binding domain (DB), while URV8 is fused to a transcriptional activation domain (TA). This has also been driven by PhyB, LOV and Cry. 
A

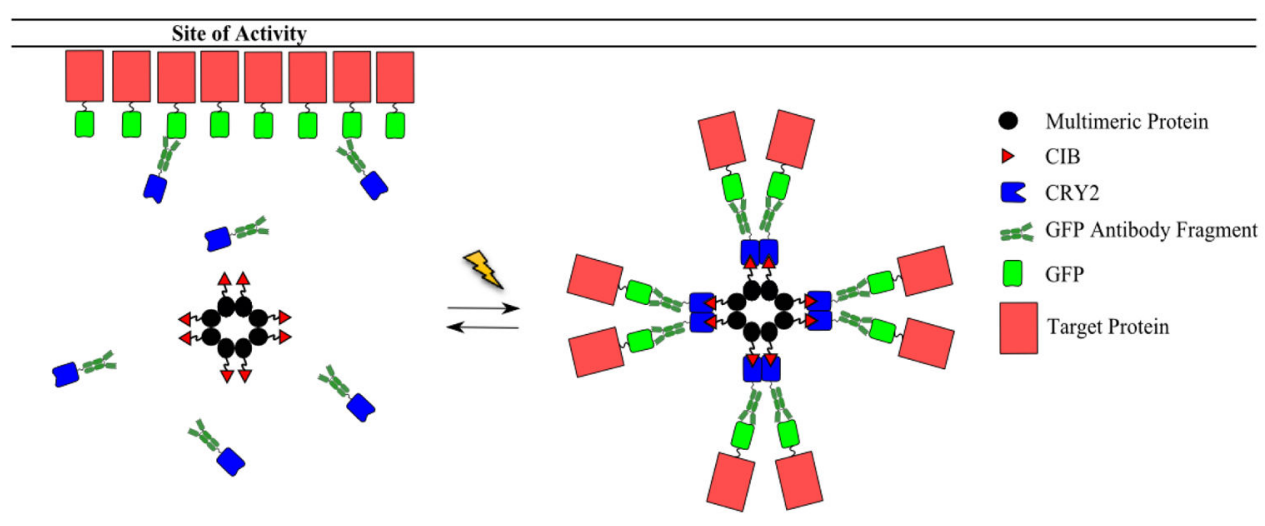

B

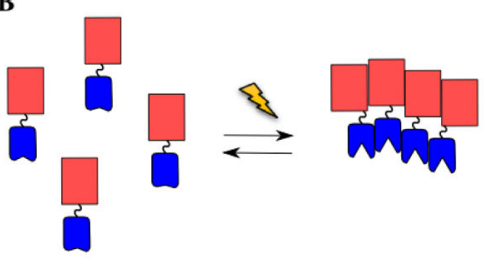

C
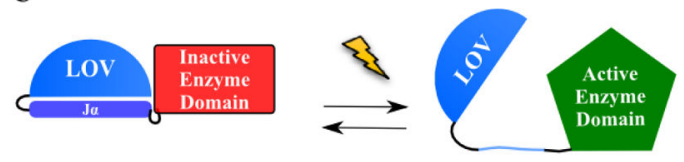

Figure 3.

Other designs for optogenetic control. A. Schematic representation of the LARIAT system. B. Light-induced clustering of CRY2. C. Light induced allosteric regulation of enzyme activity [21, 22, 40]. 